\title{
Prevalence and Associated Factors of Diabetic Retinopathy in a Russian Population. The Ural Eye and Medical Study
}

\author{
Mukharram M Bikbov' \\ Timur R Gilmanshin' \\ Rinat M Zainullin' \\ Gyulli M Kazakbaeva' \\ Ildar F Nuriyev' \\ Artur F Zaynetdinov' \\ Songhomitrra Panda- \\ Jonas $\mathbb{D}^{2}$ \\ Guzel M Bikbova' \\ Ellina M Rakhimova' \\ Iulia A Rusakova' \\ Timur A Khalimov (ID) \\ Kamila R Safiullina' \\ Albina A Fakhretdinova' \\ Azaliia M Tuliakova' \\ Ainur $\vee$ Gizzatov' \\ Jost $B$ Jonas $\mathbb{D}^{3,4}$ \\ 'Ufa Eye Research Institute, Ufa, \\ Bashkortostan, Russia; ${ }^{2}$ Privatpraxis Prof \\ Jonas und Dr Panda-Jonas, Heidelberg, \\ Germany; ${ }^{3}$ Department of \\ Ophthalmology, Medical Faculty \\ Mannheim, Heidelberg University, \\ Mannheim, Germany; ${ }^{4}$ Institute of \\ Molecular and Clinical Ophthalmology \\ Basel, Basel, Switzerland
}

Correspondence: Mukharram M Bikbov; Jost B Jonas

Tel +7 347 272-37-75; +49-622I-3929320

Email Bikbov.m@gmail.com; Jost.

Jonas@medma.uni-Heidelberg.de

\begin{abstract}
Aim: To assess prevalence and associated factors of diabetic retinopathy (DR) in a Russian population.

Methods: Out of 7328 eligible individuals, the population-based cross-sectional Ural Eye and Medical Study included 5899 (80.5\%) individuals aged 40+ years, who underwent a detailed medical and ophthalmological examination. Using ocular fundus photographs and optical coherence tomographic images, we assessed prevalence and degree of DR in 5105 participants.

Results: DR was present in 99/5105 individuals (1.9\%; 95\% confidence interval [CI]: 1.6 , $2.3)$. Its prevalence increased from $6 / 657(1.0 \%$; $95 \%$ CI: $0.2,1.6)$ in the age group of $45-50$ years to $24 / 680(3.5 \%$; $95 \% \mathrm{CI}: 2.1,4.9)$ in the age group of $65-70$ years, and decreased to $3 /$ $153(2.0 \%$; $95 \%$ CI: $0.00,4.2)$ in the age group of $80+$ years. DR prevalence within the 577 $(11.4 \%$; 95\% CI: 10.5, 12.2) individuals with diabetes was 99/577 (17.2\%; 95\% CI: 14.1 , 20.2). DR was the cause for moderate-to-severe vision impairment (best corrected visual acuity $<6 / 18$ but $\geq 3 / 60$ ) in four individuals (4/5105; 0.07\%). In multivariable analysis, higher DR prevalence was associated with higher serum glucose concentration (odds ratio [OR]: 1.30; 95\% CI: 1.20, 141), longer diabetes duration (OR: 1.06; 95\% CI: 1.02, 1.09), type of diabetes therapy (nil/diet/oral/insulin) (OR:4.19;95\% CI:3.08, 5.70), lower educational level (OR:0.81;95\% CI:0.67, 0.98), lower manual dynamometric force (OR: 0.96; 95\% CI: 0.94 , 0.99), shorter ocular axial length (OR: $0.73 ; 95 \% \mathrm{CI}: 0.56,0.96)$, and higher diastolic blood pressure (OR: 1.04; 95\% CI: 1.01, 1.06), or alternatively, higher estimated cerebrospinal fluid pressure (OR: 1.09 ; 95\% CI: 1.01, 1.18).

Conclusion: In this urban and rural Russian population aged $40+$ years, DR prevalence was relatively low $(1.9 \%$; 95\% CI: 1.6, 2.3), showed an inverted U-shaped association with age, and in a cross-sectional study design it was associated with shorter axial length and higher estimated cerebrospinal fluid pressure.
\end{abstract}

Keywords: diabetic retinopathy, cerebrospinal fluid pressure, myopia, axial length, blindness, ural eye and medical study

\section{Plain Language Summary}

A multiethnic population in Russia with an age of $40+$ years had a prevalence of diabetic retinopathy of $1.9 \%$. Individuals with shorter (hyperopic) eyes and a higher estimated cerebrospinal fluid pressure were more prone to have diabetic retinopathy.

Diabetic retinopathy (DR) belongs to the most common causes of blindness in the working-age group in Western populations. ${ }^{1}$ Meta-analyses revealed that in the year 2015 DR caused $1.06 \%$ (80\% uncertainty interval (UI) $0.15-2.38)$ of all cases of blindness 
worldwide, and $1.30 \%$ (80\% UI: $0.20-2.93)$ of all cases with moderate-to-severe visual impairment (MSVI). ${ }^{1}$ In the period from 1990 and 2015, the number of individuals affected by DRrelated blindness globally increased from 0.2 million ( $80 \%$ UI: 0 to 1.0 million) to 0.4 million ( $80 \%$ UI: 0 to 1.5 million), and the number of people affected by DR-related MSVI increased from 1.4 million ( $80 \%$ UI: 0.1 million to 5.4 million) to 2.6 million ( $80 \%$ UI: 0.2 million to 9.9 million). ${ }^{1}$

While numerous studies have addressed the prevalence of DR in various study populations from all continents, there have been diverging findings about the associations of DR with parameters not directly related to diabetes, such as ocular axial length and cerebrospinal fluid pressure. ${ }^{2-4}$ In addition, although DR is of importance for vision impairment, in particular in the middleaged population group, there has been no information available about the prevalence of DR, the frequency of DR as cause for MSVI and blindness, and the associations of DR with other ocular and general parameters in Russia. ${ }^{5}$ We therefore performed this study to assess the prevalence of DR and its frequency as cause for MSVI and blindness in a population from Russia, and to explore associations of DR with other systemic and ocular parameters. To reduce the potential bias caused by a referral of study participants, we chose a population-based recruitment of the study participants. To reduce the risk of a bias due to hidden confounding factors, we included a whole panoply of ocular and systemic variables for the assessment of the associations of DR.

\section{Methods}

In a rural region and in the capital city Ufa of the Russian republic of Bashkortostan, we conducted the populationbased Ural Eye and Medical Study in the period from 2015 to 2017. Ufa is located about $1300 \mathrm{~km}$ east of Moscow. ${ }^{6,7}$ In agreement with the Declaration of Helsinki, the Ethics Committee of the Academic Council of the Ufa Eye Research Institute approved the study design (Ethical Committee \#N 3, dated 16.3.2015), and all study participants signed an informed written consent. Inclusion criteria were an age of $40+$ years and living in the study regions.

All study participants underwent a series of examinations including a standardized interview with more than 250 questions on the socioeconomic background, smoking habits and alcohol consumption and other parameters (Tables 1-3). We measured the blood pressure, handgrip force, anthropometric and spirometric parameters, and performed a biochemical analysis of blood samples taken under fasting conditions. ${ }^{6,7}$ Diagnostic criteria for diabetes mellitus were a fasting serum glucose concentration of $\geq 7.0 \mathrm{mmol} / \mathrm{L}$ or a self-reported history of physicianbased diagnosis or therapy of diabetes mellitus.

The ophthalmologic examinations included automated refractometry (Auto-2Ref/Keratometer HRK-7000A Huvitz Co, Ltd., Gyeonggi-do, Korea), determination of best corrected visual acuity, slit lamp biomicroscopy of the anterior ocular segment as performed by a fellowshiptrained ophthalmologist, and non-contact tonometry. After inducing medical mydriasis (tropicamide $0.8 \%$ and phenylephrine $5 \%$ given twice in a 10 -minute interval), we performed a second slit lamp examination to assess the presence of pseudoexfoliation of the lens. ${ }^{7}$ We took digital photographs of the cornea and lens for the assessment of lens opacities (Topcon slit lamp and camera, Topcon Corp. Tokyo, Japan). We examined the optic disc and macula on digital $60^{\circ}$ photographs (VISUCAM 500, Carl Zeiss Meditec AG, Jena, Germany), centered on the macula. We additionally performed spectral-domain optical coherence tomography (OCT) (RS-3000, NIDEK co., Ltd., Aichi Japan). The detectability of a structure as small as a retinal micro-aneurysm on the fundus photographs was the quality criterion of fundus photographs to be included into the present study. The OCT images served for measurement of the optic nerve head and examination of the macula in search for macula edema or other signs of DR. We defined DR as suggested by the Early Treatment Diabetic Retinopathy Study Research Group. We defined vision threatening DR (VTDR) by the presence of severe non-proliferative DR, proliferative DR or clinically significant macular edema. ${ }^{8}$ We classified glaucoma, age-related macular degeneration and fundus tessellation as described previously. ${ }^{11,12}$ We estimated the cerebrospinal fluid pressure (eCSFP) using the formula of "eCSFP $=0.435 \times$ body mass index $\left(\mathrm{kg} / \mathrm{m}^{2}\right)+0.162 \times$ diastolic blood pressure $(\mathrm{mmHg})-0.181 \times$ age (years) -1.91 ". 9 We had tested this formula in a previous study in which we compared the calculated value of the CSFP with the CSFP as measured by lumbar puncture. 9,10

A team of ophthalmologists primarily assessed all fundus photographs including the OCT images. A panel of ophthalmologists (including MMB, JBJ, TRG, GMK) then re-examined those images with any fundus abnormality (including DR, glaucoma, age-related macular degeneration, retinal vein occlusions, myelinated retinal nerve fibers or any other disorder or abnormalities) and all fundus image of any participant with an intraocular pressure of $\geq 21 \mathrm{mmHg}$ or a best corrected visual acuity of lower than 0.8 (decimal) (equivalent to $20 / 25$ or logMAR 
Table I Demographic and Systemic Parameters (Mean \pm Standard Deviation) of the Study Population

\begin{tabular}{|c|c|c|c|c|}
\hline Parameter & $\begin{array}{l}\text { Total Study } \\
\text { Population }\end{array}$ & Diabetic Group & $\begin{array}{c}\text { Group with } \\
\text { Diabetic } \\
\text { Retinopathy }\end{array}$ & $\begin{array}{c}\text { Group without } \\
\text { Diabetic } \\
\text { Retinopathy }\end{array}$ \\
\hline $\mathrm{n}$ & 5105 & 577 & 99 & 478 \\
\hline Age (years) & $58.6 \pm 10.5$ & $63.5 \pm 9.6$ & $62.9 \pm 8.2$ & $63.6 \pm 9.8$ \\
\hline Sex (men/women) & $2043 / 3062$ & $267 / 310$ & $27 / 72$ & $|64 / 3| 4$ \\
\hline Region of habitation (rural/urban) & $3030 / 2075$ & $267 / 310$ & $53 / 46$ & $214 / 264$ \\
\hline Ethnicity (Russian/Non-Russian) & $1016 / 3556$ & $153 / 348$ & $26 / 68$ & $127 / 280$ \\
\hline Body height $(\mathrm{cm})$ & $164.5 \pm 8.6$ & $163.1 \pm 8.9$ & $162.0 \pm 8.2$ & $163.4 \pm 9.0$ \\
\hline Body weight (kg) & $75.7 \pm 14.5$ & $81.0 \pm 15.9$ & $79.9 \pm 15.6$ & $81.2 \pm 16.0$ \\
\hline Body mass index $\left(\mathrm{kg} / \mathrm{m}^{2}\right)$ & $28.0 \pm 5.0$ & $30.4 \pm 5.4$ & $30.4 \pm 5.3$ & $30.4 \pm 5.5$ \\
\hline Waist circumference $(\mathrm{cm})$ & $94.0 \pm 13.3$ & $100.6 \pm 13.5$ & $101.1 \pm 14.1$ & $100.4 \pm 13.3$ \\
\hline Hip circumference $(\mathrm{cm})$ & $103.9 \pm 12.5$ & $108.3 \pm 12.9$ & $107.8 \pm 11.7$ & $108.4 \pm 13.1$ \\
\hline Waist/hip circumference ratio & $0.91 \pm 0.09$ & $0.93 \pm 0.09$ & $0.94 \pm 0.09$ & $0.93 \pm 0.09$ \\
\hline Socioeconomic Score & $5.9 \pm 1.5$ & $5.7 \pm 1.6$ & $5.3 \pm 1.6$ & $5.8 \pm 1.6$ \\
\hline Level of education & $5.6 \pm 1.4$ & $5.4 \pm 1.5$ & $5.1 \pm 1.6$ & $5.5 \pm 1.5$ \\
\hline Systolic blood pressure (mm Hg) & $133.2 \pm 20.5$ & $142.5 \pm 21.7$ & $147.0 \pm 22.0$ & $141.5 \pm 21.5$ \\
\hline Diastolic blood pressure $(\mathrm{mm} \mathrm{Hg})$ & $81.9 \pm 10.4$ & $84.0 \pm 10.3$ & $87.1 \pm 11.8$ & $83.3 \pm 9.9$ \\
\hline Glucose blood concentration (mmol/L) & $5.03 \pm 1.68$ & $7.70 \pm 3.33$ & $9.64 \pm 4.34$ & $7.29 \pm 2.92$ \\
\hline High-density lipoprotein(mmol/L) & $2.32 \pm 0.89$ & $2.20 \pm 0.93$ & $2.15 \pm 1.06$ & $2.21 \pm 0.90$ \\
\hline Low-density lipoprotein (mmol/L) & $2.14 \pm 1.18$ & $1.99 \pm 1.12$ & $2.01 \pm 0.32$ & $1.99 \pm 1.08$ \\
\hline Triglycerides (mmol/L) & $1.42 \pm 0.76$ & $1.80 \pm 1.31$ & $2.02 \pm 2.03$ & $1.75 \pm 1.08$ \\
\hline Diabetes therapy (nil/diet/oral/insulin) & $4741 / 22 / 259 / 61$ & $235 / 22 / 259 / 61$ & $18 / 0 / 51 / 30$ & $217 / 22 / 208 / 3 \mid$ \\
\hline Duration of diabetes (years) & $0.54 \pm 3.08$ & $4.74 \pm 8.01$ & $9.36 \pm 11.0$ & $3.78 \pm 6.88$ \\
\hline Hearing loss score $(0-44)$ & $4.9 \pm 10.7$ & $5.3 \pm 10.9$ & $7.2 \pm 12.5$ & $4.9 \pm 10.5$ \\
\hline Depression Score (range: -4 to +15 ) & $1.2 \pm 3.8$ & $1.8 \pm 3.9$ & $2.3 \pm 4.1$ & $1.7 \pm 3.9$ \\
\hline State-Trait Anxiety Inventory score (range: -7 to 13 ) & $-0.57 \pm 3.57$ & $-0.13 \pm 3.69$ & $0.51 \pm 4.02$ & $-0.26 \pm 3.61$ \\
\hline Manual dynamometry, right hand (dekaNewton) & $30.1 \pm 11.5$ & $27.0 \pm 11.3$ & $23.9 \pm 10.6$ & $27.6 \pm 11.3$ \\
\hline Manual dynamometry, left hand (dekaNewton) & $26.5 \pm 11.1$ & $23.4 \pm 10.8$ & $21.8 \pm 9.6$ & $23.7 \pm 11.0$ \\
\hline Axial length $(\mathrm{mm})$ & $23.3 \pm 1.1$ & $23.2 \pm 1.2$ & $23.0 \pm 1.2$ & $23.2 \pm 1.2$ \\
\hline
\end{tabular}

(logarithm of the minimal angle of resolution) 0.1 ). We took the data of the eye with the severe stage of DR for further statistical analysis.

For the present study, we included all eyes for which the fundus photographs allowed the assessment of the presence and severity of DR, ie the detectability of a structure as small as a retinal micro-aneurysm. Using a software package (SPSS/Windows, 25.0, IBM-SPSS, Chicago, IL, USA), we first calculated the mean values (and 95\% confidence intervals (CI)) of the outcome parameters. In a following binary logistic regression analyses, we tested relationships between the DR prevalence and systemic and ocular parameters. We eventually performed a multivariable binary regression analysis with forward inclusion of the independent variables to assess associations between the DR prevalence and all those variables which were significantly $(P<0.05)$ correlated with the DR prevalence in the univariable analyses (Tables 2 and 3 ). In a backward selection, we then dropped, in a step-by-step manner, out of the list of independent variables all those parameters which were no longer significantly associated with the DR prevalence. We started with the parameters with the highest $P$-value. When the model eventually included only parameters with a $P$-value of $<0.05$, we added again to the model parameters which had been dropped, and we re-checked their association with the DR prevalence. In a third step, we performed a forward selection of parameters and compared the result with the findings obtained by the backward selection of independent parameters. Odds ratios (OR) and their 95\% CIs were determined.

\section{Results}

Out of a total group of 7328 eligible individuals, 5899 (80.5\%) individuals (3319 [56.3\%] women) with a mean age of $59.0 \pm 10.7$ years (range: $40-94$ years) participated in 
Table 2 Associations (Univariate Analysis) Between the Prevalence of Diabetic Retinopathy and Systemic Parameters in the Ural Eye and Medical Study

\begin{tabular}{|c|c|c|c|c|}
\hline Parameter & Interval & $\begin{array}{l}\text { Odds } \\
\text { Ratio } \\
\text { (OR) }\end{array}$ & $\begin{array}{l}95 \% \\
\text { Confidence } \\
\text { Interval of } \\
\text { OR }\end{array}$ & $P$-value \\
\hline Age & I-year intervals & 1.04 & $1.02,1.06$ & $<0.001$ \\
\hline Gender & Men/Women & 1.80 & $\mathrm{I} .15,2.8 \mathrm{I}$ & 0.01 \\
\hline Region of habitation & Rural/Urban & 1.27 & $0.85,1.90$ & 0.24 \\
\hline Ethnicity & Any other ethnicity/Russian & 1.39 & $0.88,2.19$ & 0.16 \\
\hline Body height & $\mathrm{I} \mathrm{cm}$ & 0.97 & $0.94,0.99$ & 0.004 \\
\hline Body weight & $\mathrm{kg}$ & 1.02 & $1.01,1.03$ & 0.004 \\
\hline Body mass index & $\mathrm{kg} / \mathrm{m}^{2}$ & 1.09 & $1.05,1.12$ & $<0.001$ \\
\hline Waist circumference & $\mathrm{cm}$ & 1.04 & $1.03,1.06$ & $<0.001$ \\
\hline Hip circumference & $\mathrm{cm}$ & 1.03 & I.0I, 1.04 & 0.001 \\
\hline Waist/hip circumference ratio & Ratio & 21.6 & $4.10,114.1$ & $<0.001$ \\
\hline Socioeconomic score & Score & 0.78 & $0.69,0.89$ & $<0.001$ \\
\hline Level of education & $\begin{array}{c}\text { Illiteracy/Passing 5th Grade/8th Grade/ } \\
\text { I0th Grade/I I th Grade/Specialized } \\
\text { Secondary Education/Graduates/Post } \\
\text { Graduates }\end{array}$ & 0.78 & $0.69,0.88$ & 0.001 \\
\hline Physical activity score & Score & 0.94 & $0.91,0.98$ & 0.001 \\
\hline Smoking, currently & Yes/No & 0.15 & $0.04,0.61$ & 0.008 \\
\hline Smoking, package years & Number & 0.92 & $0.85,0.99$ & 0.03 \\
\hline Alcohol consumption, any & Yes/No & 0.79 & $0.47,1.33$ & 0.37 \\
\hline In a week how many days do you eat vegetables? & Number of days & 0.89 & $0.79,1.00$ & 0.06 \\
\hline History of cardiovascular disorders including stroke & Yes/No & 2.71 & $1.80,4.08$ & $<0.001$ \\
\hline History of arthritis & Yes/No & 1.68 & $1.12,2.53$ & 0.01 \\
\hline History of low back pain & Yes/No & 1.65 & I.07, 2.54 & 0.02 \\
\hline History of thyroid disorder & Yes/No & 1.82 & $1.09,3.06$ & 0.02 \\
\hline History of falls & Yes/No & 1.79 & $1.16,2.78$ & 0.009 \\
\hline History of unconsciousness & Yes/No & 2.53 & $1.50,4.26$ & $<0.001$ \\
\hline History of menopause & Yes/No & 8.42 & $2.06,34.5$ & 0.003 \\
\hline \multicolumn{5}{|l|}{ Serum concentration of: } \\
\hline $\begin{array}{l}\text { Aspartate aminotransferase-to-Alanine aminotransferase } \\
\text { ratio }\end{array}$ & Ratio & 0.33 & $0.12,0.89$ & 0.03 \\
\hline High-density lipoproteins & $\mathrm{mmol} / \mathrm{L}$ & 0.78 & $0.60,1.01$ & 0.06 \\
\hline Low-density lipoproteins & $\mathrm{mmol} / \mathrm{L}$ & 0.90 & $0.74,1.09$ & 0.28 \\
\hline
\end{tabular}

(Continued) 
Table 2 (Continued).

\begin{tabular}{|c|c|c|c|c|}
\hline Parameter & Interval & $\begin{array}{l}\text { Odds } \\
\text { Ratio } \\
\text { (OR) }\end{array}$ & $\begin{array}{c}95 \% \\
\text { Confidence } \\
\text { Interval of } \\
\text { OR }\end{array}$ & $P$-value \\
\hline Cholesterol & $\mathrm{mmol} / \mathrm{L}$ & 1.06 & $0.98,1.15$ & 0.15 \\
\hline Triglycerides & $\mathrm{mmol} / \mathrm{L}$ & 1.56 & $1.34,1.81$ & $<0.001$ \\
\hline Glucose & $\mathrm{mmol} / \mathrm{L}$ & 1.64 & $1.54,1.74$ & $<0.001$ \\
\hline Urea & $\mathrm{mmol} / \mathrm{L}$ & 1.10 & $0.99,1.23$ & 0.07 \\
\hline Creatinine & $\mu \mathrm{mol} / \mathrm{L}$ & 0.99 & $0.98,1.001$ & 0.07 \\
\hline Leukocyte count & $10^{9}$ cells $/ \mathrm{L}$ & 1.13 & $1.00,1.27$ & 0.05 \\
\hline Monocytes & $\%$ of leukocytes & 1.07 & $1.00,1.15$ & 0.05 \\
\hline Estimated glomerular filtration rate & $30 \mathrm{~mL} / \mathrm{min} / 1.73 \mathrm{~m}^{2}$ & 1.00 & $0.99,1.01$ & 0.78 \\
\hline Diabetes mellitus, known duration & Years & 1.22 & $1.19,1.26$ & $<0.001$ \\
\hline Diabetes mellitus, type of therapy & Nil/diet/oral/insulin & 6.81 & $5.52,8.41$ & $<0.001$ \\
\hline Type of diabetes & Type I/2 & 7.81 & $6.16,9.90$ & $<0.001$ \\
\hline Blood pressure, systolic (SBP) & $\mathrm{mm} \mathrm{Hg}$ & 1.03 & $1.02,1.04$ & $<0.001$ \\
\hline Blood pressure, diastolic (DBP) & $\mathrm{mm} \mathrm{Hg}$ & 1.05 & $1.03,1.06$ & $<0.001$ \\
\hline Blood pressure, mean & $\mathrm{mm} \mathrm{Hg}$ & 1.05 & $1.03,1.06$ & $<0.001$ \\
\hline Arterial hypertension & Yes/No & 18.9 & $2.64,136$ & 0.003 \\
\hline Arterial hypertension, stage & $0-4$ & 2.37 & $1.78,3.16$ & $<0.001$ \\
\hline Ankle-brachial-index, right & ratio & 0.19 & $0.03,1.14$ & 0.07 \\
\hline Ankle-brachial-index, left & ratio & 0.21 & $0.03,1.26$ & 0.09 \\
\hline Ankle-brachial-index, highest values right and left & ratio & 0.21 & $0.04,1.30$ & 0.09 \\
\hline Hearing loss & Hearing loss score (0-44) & 1.02 & $1.00,1.03$ & 0.04 \\
\hline Depression score & $\begin{array}{l}\text { Depression score unit (range: }-4 \text { to } \\
\qquad+15)\end{array}$ & 1.07 & $1.02,1.13$ & 0.006 \\
\hline State-Trait Anxiety Inventory & $\begin{array}{l}\text { State-Trait Anxiety Inventory Score } \\
\text { (range: }-7 \text { to 13) }\end{array}$ & 1.08 & $1.03,1.14$ & 0.003 \\
\hline Manual dynamometry, right hand & dekaNewton & 0.95 & $0.93,0.97$ & $<0.001$ \\
\hline Manual dynamometry, left hand & dekaNewton & 0.96 & $0.94,0.98$ & $<0.001$ \\
\hline
\end{tabular}

the study. The study population did not differ significantly in sex and age from the Russian population as explored in the census of $2010 .^{11}$ The study population consisted of 1185 (20.1\%) Russians, 2439 (41.3\%) Tartars, 1061 (18.0\%) Bashkirs, 587 (10\%) Chuvash, 21 (0.4\%) Mari, $104(1.8 \%)$ individuals of other ethnicities, and 502 (8.5\%) individuals did not indicate their ethnic background.
The fundus photographs of $5105(86.5 \%)$ study participants $(2043(40.0 \%)$ men) were assessed. The mean age was $58.6 \pm 10.5$ years (range: $40-94$ years) and mean axial length was $23.3 \pm 1.1 \mathrm{~mm}$ (range: $19.78-32.87 \mathrm{~mm}$ ) (Table 1). The group of individuals with fundus assessment for DR and the group of participants without assessable fundus photographs was significantly younger $(58.6 \pm 10.5$ years versus $61.6 \pm 11.4$ 
Table 3 Associations (Univariate Analysis) Between the Prevalence of Diabetic Retinopathy and Ocular Parameters in the Ural Eye and Medical Study

\begin{tabular}{|c|c|c|c|c|}
\hline Parameter & Interval & Odds Ratio (OR) & 95\% Confidence Interval of OR & $P$-value \\
\hline Refractive error, spherical equivalent & Diopters & 1.09 & $0.98,1.21$ & 0.12 \\
\hline Refractive error, cylindrical value & Diopters & 0.87 & $0.68,1.12$ & 0.29 \\
\hline Axial length & $\mathrm{mm}$ & 0.71 & $0.56,0.89$ & 0.004 \\
\hline Corneal refractive power & Diopters & 1.08 & $0.94,1.24$ & 0.29 \\
\hline Central corneal thickness & $\mu \mathrm{m}$ & 1.01 & $1.002,1.02$ & 0.008 \\
\hline Corneal volume & $\mathrm{mm}^{3}$ & 1.09 & $1.03,1.15$ & 0.002 \\
\hline Anterior chamber depth & $\mathrm{mm}$ & 0.74 & $0.45,1.22$ & 0.24 \\
\hline Anterior chamber volume & $\mu \mathrm{L}$ & 0.99 & $0.98,0.999$ & 0.01 \\
\hline Anterior chamber angle & Degree & 0.98 & $0.95,1.01$ & 0.23 \\
\hline Lens thickness & $\mathrm{mm}$ & 2.11 & $1.26,3.53$ & 0.005 \\
\hline Intraocular pressure & $\mathrm{mmHg}$ & 1.12 & $1.07,1.18$ & $<0.001$ \\
\hline Nuclear cataract degree & Grade & 1.20 & $0.95,1.54$ & 0.13 \\
\hline Nuclear cataract, presence & Yes/No & 1.29 & $0.80,2.08$ & 0.30 \\
\hline Cortical cataract, degree & Percentage & 1.00 & $0.97,1.03$ & 0.88 \\
\hline Cortical cataract, presence & Yes/No & 1.28 & $0.67,2.45$ & 0.46 \\
\hline Any cataract & Yes/No & 1.16 & $0.75,1.80$ & 0.50 \\
\hline Status after cataract surgery & Yes/No & 3.70 & $1.88,7.28$ & 0.001 \\
\hline Age-related macular degeneration, any & Yes/No & 0.60 & $0.24,1.49$ & 0.27 \\
\hline Age-related macular degeneration, early & Yes/No & 0.69 & $0.25,1.9 !$ & 0.48 \\
\hline Age-related macular degeneration, intermediate & Yes/No & 0.00 & 0.00 & 0.99 \\
\hline Age-related macular degeneration, late & Yes/No & 2.09 & $0.28,15.5$ & 0.47 \\
\hline Fundus tessellation, macula region & Grade & 1.19 & $0.94,1.52$ & 0.15 \\
\hline Fundus tessellation, peripapillary region & Grade & 1.15 & $0.93,1.43$ & 0.21 \\
\hline Retinal thickness (total), fovea & $\mu \mathrm{m}$ & 1.006 & $1.003,1.008$ & $<0.001$ \\
\hline Retinal thickness (total), $300 \mu \mathrm{m}$ temporal to the fovea & $\mu \mathrm{m}$ & 1.006 & $1.003,1.009$ & $<0.001$ \\
\hline Retinal thickness (total), $300 \mu \mathrm{m}$ nasal to the fovea & $\mu \mathrm{m}$ & 1.005 & $1.002,1.007$ & $<0.001$ \\
\hline \multirow[t]{2}{*}{ Retinal nerve fiber layer thickness } & \multirow[t]{2}{*}{$\mu \mathrm{m}$} & \multirow[t]{2}{*}{1.00} & $0.99,1.02$ & \multirow[t]{2}{*}{0.99} \\
\hline & & & I & \\
\hline Pseudoexfoliation of the lens & Yes/No & 0.76 & $0.19,3.11$ & 0.70 \\
\hline Glaucoma & Yes/No & 1.93 & $0.78,4.77$ & 0.16 \\
\hline Glaucoma stage & $0-5$ & 1.30 & $0.92,1.84$ & 0.14 \\
\hline Open-angle glaucoma & Yes/No & 1.49 & $0.47,4.71$ & 0.50 \\
\hline Angle-closure glaucoma & Yes/No & 3.18 & $0.75,13.4$ & 0.12 \\
\hline Myopic maculopathy, stage & $0-4$ & 0.70 & $0.29,1.66$ & 0.41 \\
\hline
\end{tabular}




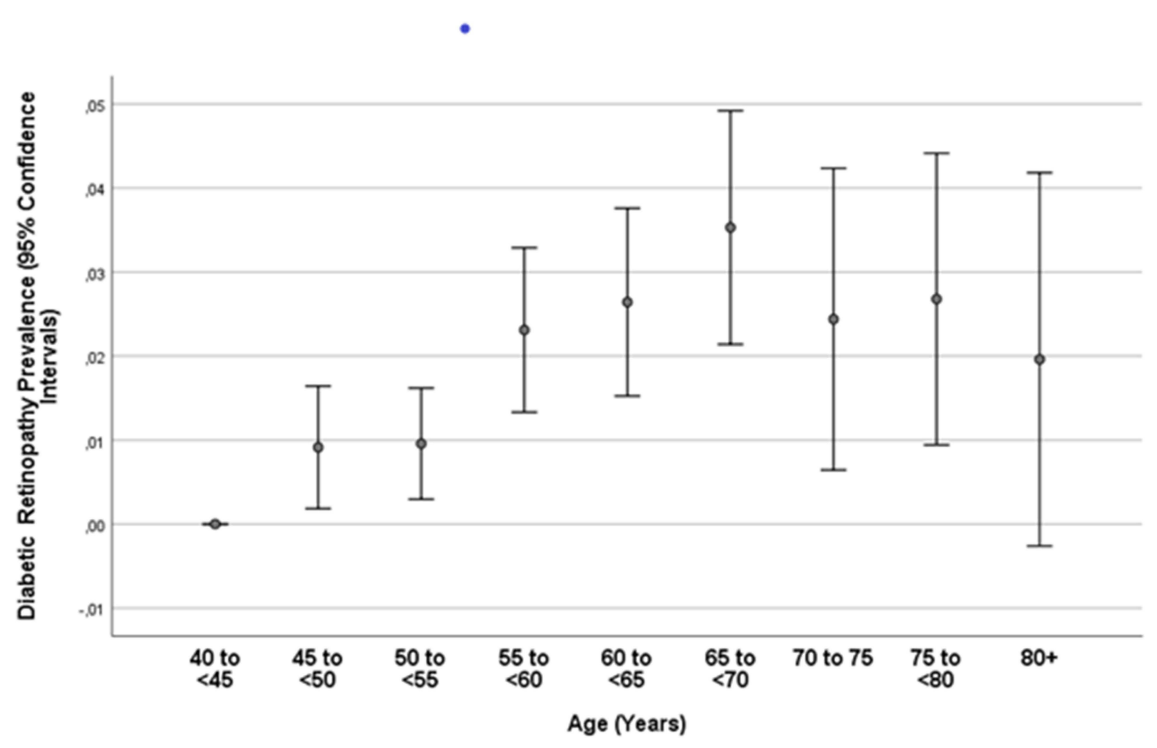

Figure I Prevalence of diabetic retinopathy in the Ural Eye and Medical Study.

years; $P<0.001$ ), showed a significantly higher proportion of women $(3062 / 5105$ or $60 \%$ versus $257 / 794$ or $32.4 \%$; $P<0.001)$, and had a shorter axial length $(23.3 \pm 1.1 \mathrm{~mm}$ versus $23.4 \pm 1.2 \mathrm{~mm} ; P=0.04)$.

DR in the worse eye per study participant was present in 99/5105 individuals $(1.9 \% ; 95 \%$ CI: $1.6,2.3)$. In the total study population, the DR prevalence increased from $6 / 657$ $(1.0 \%$; $95 \%$ CI: $0.2,1.6)$ in the age group of 45 to $<50$ years to $24 / 680(3.5 \%$; $95 \% \mathrm{CI}: 2.1,4.9)$ in the age group of 65 to $<70$ years, and decreased to $9 / 336(2.7 \%$; $95 \% \mathrm{CI}: 0.9,4.4)$ in the age group of 75 to $<80$ years and to $3 / 153(2.0 \%$; $95 \% \mathrm{CI}$ : $0.00,4.2$ ) in the age group of $80+$ years (Figure 1).

Among the 577 (11.4\%; 95\% CI: 10.5, 12.2) individuals with diabetes in the study population, 99 individuals had DR (17.2\%; 95\% CI: 14.1, 20.2). Among the 99 individuals with DR, 65 (65.7\%) participants had mild non-proliferative DR, 25 (25.3\%) individuals had moderate non-proliferative DR, 5 (5.1\%) participants had severe non-proliferative DR, and 4 individuals $(4.0 \%)$ had proliferative DR. Twelve (12\%) individuals had diabetic macular edema, and 18 individuals $(18.2 \%, 95 \%$ CI: $10.5,25.9)$ had VTDR. In the group of individuals with diabetes, the DR prevalence slightly increased from $6 / 33$ (18.2\%; 95\% CI: $4.3,32.1)$ in the age group of 45 to $<50$ years to $21 / 100(21.0 \%$; $95 \%$ CI: $12.9,29.1)$ in the age group of 55 to $<60$ years, and it decreased to $9 / 63(14.3 \%$; $95 \%$ CI: 5.4, 23.2) age group of 75 to $<80$ years and to $3 / 26(11.5 \%$; $95 \%$ CI: $0.00,24.7)$ in the age group of $80+$ years.

Among the whole group of study participants, DR was the cause for MSVI (defined as best corrected visual acuity $<6 / 18$ but $\geq 3 / 60$ inclusive in the better eye or in binocular viewing) in four individuals (4/5105 or $0.07 \%$ ), and cause for blindness in none of the study participants. Among the 122 individuals with MSVI in the whole study population, four $(3.3 \%)$ individuals had MSVI due to DR.

Including the whole study population, a higher DR prevalence was significantly associated with various systemic and ocular parameters (Tables 2 and 3). In the multivariable analysis, we first adjusted for systemic parameters. We included as independent parameters all those variables which were associated with the prevalence of DR in the univariate analysis $(P<0.05)$. Due to collinearity, we dropped the parameters of body weight, hip and waist circumference, as well as prevalence and stage of arterial hypertension. Due to a lack of significance, we dropped step by step the parameters of history of arthritis $(P=0.88)$, depression score $(P=0.94)$, body height $(P=0.79)$, anxiety score $(P=0.60)$, history of low back pain $(P=0.64)$ and thyroid disorders $(P=0.58)$, aspartate aminotransferase-to-alanine aminotransferase ratio $(P=0.55)$, history of falls $(P=0.52)$, systolic blood pressure $(P=0.50)$, body mass index $(P=0.65)$, hearing loss score $(P=0.52)$, physical activity score $(P=0.40)$, history of cardiovascular disease $(P=0.32)$ and unconsciousness $(P=0.06)$, sex $(P=$ $0.17)$, smoking $(P=0.16)$, serum concentration of triglycerides $(P=0.26)$, type of diabetes $(P=0.16)$, and age $(P=0.06)$. We then added all ocular parameters which were significantly associated with the DR prevalence in the univariate analysis, to the list of independent variables. Due to the lack of significance, we then dropped the parameters of corneal volume $(P=0.43)$, anterior chamber volume $(P=0.85)$, intraocular pressure $(P=0.52)$, previous cataract surgery $(P=0.99)$, and 
Table 4 Associations (Multivariable Binary Regression Analysis) Between the Prevalence of Diabetic Retinopathy and Systemic and Ocular Parameters in the Ural Eye and Medical Study

\begin{tabular}{|l|c|c|c|}
\hline Parameter & Odds Ratio (OR) & 95\% Confidence Interval of OR & P-value \\
\hline Serum concentration of glucose (mmol/L) & 1.30 & $1.20,1.41$ & $<0.001$ \\
Diabetes duration (years) & 1.06 & $1.02,1.09$ & 0.003 \\
Diabetes therapy (nil/diet/oral/insulin) & 4.19 & $3.08,5.70$ & $<0.001$ \\
Level of education & 0.81 & $0.67,0.98$ & 0.03 \\
Manual dynamometric force (dekaNewton) & 0.96 & $0.94,0.99$ & 0.005 \\
Diastolic blood pressure (mm Hg) & 1.04 & $1.01,1.06$ & 0.009 \\
Ocular axial length (mm) & 0.73 & $0.56,0.96$ & 0.03 \\
Central corneal thickness $(\mu \mathrm{m})$ & 1.01 & $1.004,1.02$ & 0.002 \\
\hline
\end{tabular}

lens thickness $(P=0.31)$. In the final model, a higher DR prevalence was associated with higher serum concentration of glucose, longer known diabetes duration, type of diabetes therapy (nil/diet/oral/insulin), lower level of education, higher diastolic blood pressure, lower manual dynamometric force, shorter axial length, and thicker central corneal thickness (Table 4). If we replaced the parameter of diastolic blood pressure with the parameter of eCSFP (mean: $12.9 \pm 3.5$ $\mathrm{mmHg}$ ), a higher eCSFP was significantly associated with higher DR prevalence (OR: 1.09; 95\% CI: 1.01, 1.18). If we added in a step-by-step manner to the list of independent variables the parameters of region of habitation $(P=0.79)$, serum concentrations of triglycerides $(P=0.28)$, total bilirubin $(P=0.60)$, high-density lipoproteins $(P=0.36)$ and lowdensity lipoproteins $(P=0.67)$, and the ankle-brachial pressure index $(P=0.34)$, none of these parameters was significantly associated with the DR prevalence.

If only the study participants with diabetes were included into the statistical analysis, similar results were obtained. A higher DR prevalence was associated with higher serum concentration of glucose, longer known diabetes duration, type of diabetes therapy (nil/diet/oral/insulin), lower level of education, higher diastolic blood pressure, lower manual dynamometric force, shorter axial length, and thicker central corneal thickness (Table 5). If we replaced the parameter of diastolic blood pressure with the parameter of eCSFP (mean: $13.4 \pm 3.7 \mathrm{mmHg}$ ), the association with a higher DR prevalence was marginal (OR: $1.07 ; 95 \% \mathrm{CI}$ : 0.99, 1.16; $P=0.08)$. If we added in a step-by-step manner to the list of independent variables the parameters of region of habitation $(P=0.48)$, serum concentrations of triglycerides $(P=0.37)$, total bilirubin $(P=0.35)$, high-density lipoproteins $(P=0.78)$ and lowdensity lipoproteins $(P=0.722)$, and the ankle-brachial pressure index $(P=0.25)$, none of these parameters was significantly associated with the DR prevalence.

\section{Discussion}

The findings obtained in our study can be compared with observations made in other study populations. The DR prevalence of $17.2 \%$ found in our study population was lower than the figure in a previous meta-analysis, in which Yau et al reported on an overall global DR prevalence of $34.6 \%$ within diabetic individuals, with a prevalence of $6.96 \%$ for proliferative DR, $6.81 \%$ for diabetic macular edema, and $10.2 \%$ for

Table 5 Associations (Multivariable Binary Regression Analysis) Between the Prevalence of Diabetic Retinopathy and Systemic and Ocular Parameters in the Diabetic Subgroup of the Ural Eye and Medical Study

\begin{tabular}{|l|c|c|c|}
\hline Parameter & Odds Ratio (OR) & 95\% Confidence Interval of OR & P-value \\
\hline Serum concentration of glucose (mmol/L) & 1.23 & $1.14,1.33$ & $<0.00 \mathrm{I}$ \\
Diabetes duration (years) & 1.06 & $1.03, \mathrm{I} .10$ & $0.00 \mathrm{I}$ \\
Diabetes therapy (nil/diet/oral/insulin) & 1.85 & $1.3 \mathrm{I}, 2.6 \mathrm{I}$ & $0.00 \mathrm{I}$ \\
Level of education & 0.82 & $0.67,0.996$ & 0.045 \\
Manual dynamometric force (dekaNewton) & 0.97 & $0.94,0.998$ & 0.03 \\
Diastolic blood pressure (mm Hg) & 1.04 & $1.01,1.06$ & 0.01 \\
Ocular axial length (mm) & 0.73 & $0.55,0.98$ & 0.035 \\
Central corneal thickness $(\mu \mathrm{m})$ & 1.01 & $1.002,1.02$ & 0.02 \\
\hline
\end{tabular}


VTDR. ${ }^{12}$ Studies from Singapore and other sites reported similar figures. ${ }^{13-15}$ In contrast, the Korea National Health and Nutrition Examination Survey 2008-2011, the study conducted by Rodriguez-Poncelas et al in Spain on 109,000 patients with type 2 diabetes, and the Australian Diabetes, Obesity and Lifestyle study found similar figures as in our study population. ${ }^{16-18}$ The DR prevalence in the study population and within the group of diabetic patients in our study was higher than in the Central India Eye and Medical Study with an overall DR prevalence of $0.33 \%$ and a DR prevalence of $9.6 \%$ in the diabetic group. ${ }^{19}$ Reasons for the discrepancies between the studies may be differences in the infrastructural development of the study regions, lifestyle of the study populations, study period (with different treatment regimens available, such as intravitreal medication), and in the availability of major medical services. In particular, a low quality of the medical infrastructure might have led to a shortened life expectancy of diabetic patients in underdeveloped regions preventing the development of DR.

As in our study, a higher prevalence of DR in the previous investigations correlated with longer diabetes duration, higher serum glucose concentration or a higher $\mathrm{HbAlc}$ value, and higher blood pressure. ${ }^{12}$ In Singapore, Indian ethnicity was an additional risk factor, while higher diastolic blood pressure, higher serum concentration of total cholesterol and lowdensity lipoprotein cholesterol correlated with lower odds of any DR. ${ }^{13}$ The associations between DR prevalence and body mass index were inconclusive. To cite examples, in the Korea National Health and Nutrition Examination Survey 20082011, the prevalence of any DR correlated with a lower body mass index in a multivariable analysis. ${ }^{16}$ In contrast, a higher body mass index (BMI) correlated with a higher prevalence of DR in the studies by Durani and van Leiden, while other investigations did not find any significant relationships between BMI and DR prevalence. ${ }^{20-22}$ In our study population, BMI was not significantly related with the DR prevalence in the multivariable model. In a recent meta-analysis of studies on Asian adults with diabetes, obesity showed a significant inverse association with the prevalence of DR as a whole and with the prevalence of VTDR. ${ }^{23}$ In continuous analysis, BMI had a significant inverse association with the DR prevalence as a whole and with the prevalence of VTDR. ${ }^{23}$ Overweight did not show a significant association with the DR prevalence. ${ }^{23}$ Reasons for the discrepancies between study populations in the association between DR and BMI or obesity include a survival bias, since individuals with both, obesity and DR, might have died earlier, leaving less obese patients with DR survive and be included in a study. Other factors may be the possibility that a lower BMI may reflect a more advanced, poorly controlled stage of diabetes; the possibility that overweight/obese persons with diabetes may have a higher prevalence of comorbid conditions, may have presented earlier and medically treated better, thus preventing the development of DR; the genetic predisposition to type 2 diabetes being stronger in lean diabetic patients than obese type 2 diabetics; and that BMI does not distinguish between muscle mass and fat mass, particularly in persons with a BMI within the normal range.

The association between DR prevalence and age showed in our study population an inverted U-shape, with an increase to the age group of 65 to $<70$ years and a decrease beyond that point (Figure 1). Subsequently, DR prevalence was not related with age in the multivariable model. A similar observation was made in the Central India Eye and Medical Study in which the DR prevalence increased up to the age group of 60-64 years, and decreased in the older age groups. ${ }^{19}$ In the Singapore Malay Eye Study, older age (and a higher total cholesterol serum concentration) was associated with a lower DR prevalence. $^{24}$ The discrepancy between the results of the various studies may be due to the non-linear association between DR prevalence and age. The decrease of the DR prevalence with older age may be due to a survival effect. In our multivariable model, DR prevalence was not associated with the serum creatinine concentration or chronic kidney disease, nor with the serum bilirubin concentration. It is in contrast to the results of the Korea National Health and Nutrition Examination Survey 2008-2010, in which the statistical analysis revealed a correlation between chronic kidney disease and DR prevalence. It is in contrast to a study by Yasuda et al who reported on an association between higher DR prevalence and lower serum bilirubin concentrations. ${ }^{25,26}$

Interestingly, a higher DR prevalence in our study population correlated with shorter axial length (Table 4). The Beijing Eye Study, the Singapore Malay Eye Study, the Kailuan Eye Study and other investigations (Royal Victorian Eye and Ear Hospital, Victoria, Australia) reported on similar observations. ${ }^{2,3,24,27}$ In the Kailuan Eye Study, DR prevalence decreased by $19 \%(95 \%$ CI: 5,30$)$ for each millimeter increase in axial length after adjusting for systemic factors. ${ }^{27}$ The reasons for the protective effect of longer axial length against DR have remained elusive so far. Investigations of individuals without macular or retinal diseases showed that the intraocular concentration of vascular endothelial growth factor (VEGF) was significantly lower in eyes with longer axis or more myopic refractive error. ${ }^{28}$ The larger intraocular volume in the eyes with longer axial length might have diluted the intraocular VEGF concentration as the cause for the lower DR 
prevalence in these eyes. Interestingly, the prevalence of another intraocular VEGF-related disease, ie, age-related macular degeneration, also was lower in eyes with longer axial length. ${ }^{29}$ Additional causes for the reduced intraocular VEGF concentration in longer eyes may be a higher fluidity of the vitreous body. It may lead to a faster turnover of VEGF. The globally increasing prevalence of axial myopia may partially compensate for the influence of the prognosticated increase in the prevalence of obesity and increase in life expectancy of patients with diabetes as risk factors for DR. ${ }^{30}$

The observed relationship between a higher DR prevalence and a higher eCSFP may be of particular interest. Since the central retinal vein passes through the optic nerve head, the distal part of the optic nerve and through the orbital cerebrospinal fluid space, the intraocular venous blood pressure should be at least as high as the orbital CSFP is. Correspondingly, studies have shown that an increased CSFP correlated with an increased intraocular retinal venous pressure as measured by ophthalmodynamometry $^{31}$ that prevalence and incidence of retinal vein occlusions as another hemorrhagic retinopathy with retinal hemorrhages, lipid exudation and venous dilatation is associated with an increased eCSFP, ${ }^{32}$ and that an increased brain pressure can be associated with retinal hemorrhages, as in the case of an acute subarachnoidal hemorrhage (Terson's syndrome). The elevated retinal vein pressure in eyes with DR may explain the venous dilatation typical for DR, and the increased retinal vein pressure may lead in a retrograde manner to an increased capillary pressure in diabetic eyes. The elevated capillary pressure in association with a diabetes-related capillary wall alteration may lead to the increased prevalence of retinal hemorrhages and retinal exudation in eyes with DR. The association between DR prevalence and eCSFP may also explain why an increased arterial blood pressure is associated with a dilation of the retinal veins in diabetic eyes, since a higher arterial blood pressure is associated with a higher CSFP. $^{33}$ The association might also explain why lowering of arterial blood pressure can have a beneficial effect on DR.

Interestingly, a higher DR prevalence was associated with a lower grip strength in the multivariable analysis (Table 4). It agrees with the results obtained in the Prospective Urban Rural Epidemiology (PURE) study in which a lower grip strength was correlated with a higher rate of all-cause death, cardiovascular death, and cardiovascular disease. ${ }^{34}$ DR prevalence was not associated with the ankle-brachial pressure index in the multivariable model in our study. It may suggest that DR as a micro-angiopathy is not markedly associated with diabetesrelated changes in the large blood vessels. In the recent "No Blind Study", a U-shaped correlation has been described between the high-density lipoprotein serum concentration and the DR prevalence. ${ }^{35}$ In our study, a higher DR prevalence was marginally associated with a greater high-density lipoprotein serum concentration (OR: $0.78 ; 95 \% \mathrm{CI}: 0.60,1.01 ; P=$ $0.06)$. Future studies may explore such a correlation more in detail.

The factors associated with a higher DR prevalence as found in our and previous studies are of practical importance, since they may allow a targeted screening for patients with undetected DR. In that context, a lesson learnt from the COVID-19 pandemic may be that telemedicine in virtual clinics brings the retinal specialists closer to peripheral clinical centers, allowing a screening and follow-up of DR. ${ }^{36,37}$

Potential limitations of our study should be mentioned. First, the value of an epidemiological investigation is profoundly connected with the rate of participation and how much the study area and study population are representative for the region and population the study aimed at. In our investigation with a participation rate of $80.5 \%$ of the eligible population, a major bias in the inclusion of the study participants may appear less likely. The study areas were typical for Southern Russia with respect to its demography, geography and climate. The fraction of Russians in our study population and in the study regions was lower than in North-Western Russia and Central Russia. To address that issue, we assessed the DR prevalence in dependence of the ethnic background and did not detect a significant correlation in the multivariable analysis. Second, the group of participants with available fundus photographs for the assessment of DR as compared to the individuals without such fundus photographs were significantly younger, showed a higher proportion of women and had a shorter axial length. Since any fundus lesion such as DR can only be assessed if the optical media of the eye are clear, any eye with an advanced cataract and DR will not have been included in our study. The dependence of the assessment of the ocular fundus on the clarity of the optic media may thus have led to an underestimation of the DR prevalence in our study population, in particular since the prevalence of cataract in our study population was higher than in the populations of other investigations. Third, the observed association between DR prevalence of eCSFP markedly depends on the formula which was used to calculate the eCFSP. Fourth, one of the components of the formula of the eCSFP, ie diastolic blood pressure, was by itself correlated with DR. It was thus not possible to distinguish between a direct effect of CSFP on the DR prevalence and a secondary effect by an elevated diastolic blood pressure. Such a question may be addressed in a study comparing patients with DR and who have elevated or normal 
CSFP as measured by direct lumbar puncture. Such a design is however not possible for a population-based investigation. Fifth, the sample size of the group of patients with diabetic retinopathy ( $\mathrm{n}=99 / 5105$ individuals or $1.9 \%$ ) was relatively low for a detailed statistical analysis. This however is a weakness of any population-based study that, in relationship to the prevalence of the disease, the group of patients with the disease can be relatively small. Sixth, the definition of diabetes applied in our population-based study used criteria different from those used in hospital-based studies or in clinical routine. In the latter, usually four criteria have been applied namely a random glycemia $\geq 200 \mathrm{mg} / \mathrm{dL}$ with clinical signs, an $\mathrm{HbA1c}$ value of $\geq 6.5 \%$, glycemia $\geq 200 \mathrm{mg} / \mathrm{dL}$ two hours after an oral glucose tolerance test, and a fasting blood glucose $\geq 126 \mathrm{mg} / \mathrm{dl}$. In addition, a pathological laboratory value should be recorded twice. The differences in the definition of diabetes may have led to an underestimation of the number of participants with diabetes in our study. The strengths of our study include that it was the first population-based study from Russia on the DR prevalence, the relatively high number of study participants, and the variety of ocular and systemic parameters which were assessed and included into the statistical analysis.

In conclusion, in our typical, ethnically mixed, urban and rural Russian population aged $40+$ years, DR prevalence was relatively low $(1.9 \%$; 95\%: 1.6, 2.3), showed an inverted U-shaped association with age, and was associated with shorter axial length and higher estimated cerebrospinal fluid pressure. Future longitudinal studies may address the potential role that ocular axial length and cerebrospinal fluid pressure might play in the etiology of DR.

\section{Patent Application}

European patent application: WO 2021/198369 A1; PCT/ EP2021/058500: Agents for the use in the therapeutic or prophylactic treatment of retinal pigment epithelium associated diseases.

\section{Acknowledgments}

The abstract of this paper was presented at the Annual Meeting of the Association for Research in Vision and Ophthalmology ARVO 2019 as a poster with interim findings.

\section{Author Contributions}

All authors made a significant contribution to the work reported, whether that is in the conception, study design, execution, acquisition of data, analysis and interpretation, or in all these areas; took part in drafting, revising or critically reviewing the article; gave final approval of the version to be published; have agreed on the journal to which the article has been submitted; and agree to be accountable for all aspects of the work.

\section{Funding}

There is no funding to report.

\section{Disclosure}

Jost B. Jonas: Patent holder with Biocompatibles UK Ltd. (Farnham, Surrey, UK) (Title: Treatment of eye diseases using encapsulated cells encoding and secreting neuroprotective factor and/or anti-angiogenic factor (Patent number: 20120263794); Jost B. Jonas and Songhomitra Panda-Jonas: European patent EP 3 271392, JP 2021-119187, and US 11008385: gents for use in the therapeutic or prophylactic treatment of myopia or hyperopia. The authors report no other conflicts of interest in this work.

\section{References}

1. Leasher JL, Bourne RR, Flaxman SR, et al. Global estimates on the number of people blind or visually impaired by diabetic retinopathy: a meta-analysis from 1990 to 2010. Diabetes Care. 2016;39:1643-1649. doi: $10.2337 / \mathrm{dc} 15-2171$

2. Xie XW, Xu L, Wang YX, Jonas JB. Prevalence and associated factors of diabetic retinopathy. The Beijing Eye Study 2006. Graefes Arch Clin Exp Ophthalmol. 2008;246:1519-1526. doi:10.1007/s00417-0080884-6

3. Lim LS, Lamoureux E, Saw SM, Tay WT, Mitchell P, Wong TY. Are myopic eyes less likely to have diabetic retinopathy. Ophthalmology. 2010;117:524-530. doi:10.1016/j.ophtha.2009.07.044

4. Jonas JB, Wang N, Xu J, et al. Diabetic retinopathy and estimated cerebrospinal fluid pressure. The Beijing Eye Study 2011. PLoS One. 2014;9:e96273. doi:10.1371/journal.pone.0096273

5. Steinmetz JD, Bourne RRA, Briant PS; GBD 2019 Blindness and Vision Impairment Collaborators; Vision Loss Expert Group of the Global Burden of Disease Study. Causes of blindness and vision impairment in 2020 and trends over 30 years, and prevalence of avoidable blindness in relation to VISION 2020: the right to sight: an analysis for the Global Burden of Disease Study. Lancet Glob Health. 2021;9:e144-e160. doi:10.1016/S2214-109X(20)30489-7

6. Bikbov MM, Gilmanshin TR, Kazakbaeva GM, et al. Prevalence of myopic maculopathy among adults in a Russian population. JAMA Netw Open. 2020;3:e200567. doi:10.1001/jamanetworkopen.2020.0567

7. Bikbov MM, Fayzrakhmanov RR, Kazakbaeva GM, et al. Prevalence, awareness and control of diabetes in Russia: the Ural Eye and Medical Study on adults aged 40+ years. PLoS One. 2019;14:e0215636. doi:10.1371/journal.pone. 0215636

8. Early Treatment Diabetic Retinopathy Study Research Group. Grading diabetic retinopathy from stereoscopic color fundus photographs - an extension of the modified Airlie House classification. ETDRS report number 10. Ophthalmology. 1991;98:786-806. doi:10.1016/S0161-6420(13) 38012-9

9. Xie X, Zhang X, Fu J, et al. Noninvasive intracranial pressure estimation by orbital subarachnoid space measurement: the Beijing Intracranial and Intraocular Pressure (iCOP) study. Crit Care. 2013;17:R162. doi:10.1186/cc12841 
10. Fleischman D, Bicket AK, Stinnett SS, et al. Analysis of cerebrospinal fluid pressure estimation using formulae derived from clinical data. Invest Ophthalmol Vis Sci. 2016;57:5625-5630. doi:10.1167/ iovs.16-20119

11. Google. Public Data. Available from: https://www.google.com/public data/explore?ds $=\mathrm{z} 5567 \mathrm{oe} 244 \mathrm{~g} 0 \mathrm{ot} \_\&$ met_y=population\&idim $=$ city_ proper $\% 3 \mathrm{~A} 036580 \% 3 \mathrm{~A} 035310 \% 3 \mathrm{~A} 035240 \& \mathrm{hl}=\mathrm{en} \& \mathrm{dl}=\mathrm{en}$. Accessed November 19, 2021.

12. Yau JW, Rogers SL, Kawasaki R, et al. Global prevalence and major risk factors of diabetic retinopathy. Diabetes Care. 2012;35:556-564. doi:10.2337/dc11-1909

13. Tan GS, Gan A, Sabanayagam C, et al. Ethnic differences in the prevalence and risk factors of diabetic retinopathy: the Singapore epidemiology of eye diseases study. Ophthalmology. 2018;125:529-536. doi:10.1016/j.ophtha.2017.10.026

14. Keel S, Xie J, Foreman J, van Wijngaarden P, Taylor HR, Dirani M. The prevalence of diabetic retinopathy in Australian adults with self-reported diabetes: the National Eye Health Survey. Ophthalmology. 2017;124:977-984. doi:10.1016/j.ophtha.2017.02.004

15. Li JQ, Welchowski T, Schmid M, et al. Prevalence, incidence and future projection of diabetic eye disease in Europe: a systematic review and meta-analysis. Eur $J$ Epidemiol. 2020;35:11-23. doi:10.1007/s10654-019-00560-z

16. Jee D, Lee WK, Kang S. Prevalence and risk factors for diabetic retinopathy: the Korea National Health and Nutrition Examination Survey 2008-2011. Invest Ophthalmol Vis Sci. 2013;54:6827-6833. doi:10.1167/iovs.13-12654

17. Rodriguez-Poncelas A, Miravet-Jiménez S, Casellas A, et al. Prevalence of diabetic retinopathy in individuals with type 2 diabetes who had recorded diabetic retinopathy from retinal photographs in Catalonia (Spain). $\quad B r \quad J$ Ophthalmol. 2015;99:1628-1633. doi:10.1136/bjophthalmol-2015-306683

18. Tapp RJ, Shaw JE, Harper CA, et al. The prevalence of and factors associated with diabetic retinopathy in the Australian population. Diabetes Care. 2003;26:1731-1737. doi:10.2337/diacare.26.6.1731

19. Jonas JB, Nangia V, Khare A, et al. Prevalence and associated factors of diabetic retinopathy in rural Central India. Diabetes Care. 2013;36:e69. doi:10.2337/dc12-2377

20. Dirani M, Xie J, Fenwick E, et al. Are obesity and anthropometry risk factors for diabetic retinopathy? The diabetes management project. Invest Ophthalmol Vis Sci. 2011;52:4416-4421. doi:10.1167/iovs.11-7208

21. van Leiden HA, Dekker JM, Moll AC, et al. Blood pressure, lipids, and obesity are associated with retinopathy: the Hoorn study. Diabetes Care. 2002;25:1320-1325. doi:10.2337/diacare.25.8.1320

22. Klein R, Klein BE, Moss SE. Is obesity related to microvascular and macrovascular complications in diabetes? The Wisconsin Epidemiologic Study of Diabetic Retinopathy. Arch Intern Med. 1997;157:650-656. doi:10.1001/archinte.1997.00440270094008

23. Sabanayagam C, Sultana R, Banu R, et al. Association between body mass index and diabetic retinopathy in Asians: the Asian Eye Epidemiology Consortium (AEEC) study. Br J Ophthalmol. 2021; bjophthalmol-2020-318208. doi:10.1136/bjophthalmol-2020-318208
24. Wong TY, Cheung N, Tay WT, et al. Prevalence and risk factors for diabetic retinopathy: the Singapore Malay Eye Study. Ophthalmology. 2008;115:1869-1875. doi:10.1016/j.ophtha.2008.05.014

25. Park YH, Shin JA, Han JH, Park YM, Yim HW. The association between chronic kidney disease and diabetic retinopathy: the Korea National Health and Nutrition Examination Survey 2008-2010. PLoS One. 2015;10:e125338. doi:10.1371/journal.pone.0125338

26. Yasuda M, Kiyohara Y, Wang JJ, et al. High serum bilirubin levels and diabetic retinopathy: the Hisayama Study. Ophthalmology. 2011;118:1423-1428. doi:10.1016/j.ophtha.2010.12.009

27. Wang Q, Wang YX, Wu SL, et al. Ocular axial length and diabetic retinopathy: the Kailuan Eye Study. Invest Ophthalmol Vis Sci. 2019;60:3689-3695. doi:10.1167/iovs.19-27531

28. Jonas JB, Tao Y, Neumaier M, Findeisen P. VEGF and refractive error. Ophthalmology. 2010;117:2234. doi:10.1016/j.ophtha.2009.12.006

29. Ikram MK, van Leeuwen R, Vingerling JR, Hofman A, de Jong PT. Relationship between refraction and prevalent as well as incident age-related maculopathy: the Rotterdam Study. Invest Ophthalmol Vis Sci. 2003;44:3778-3782. doi:10.1167/iovs.03-0120

30. Holden BA, Fricke TR, Wilson DA, et al. Global prevalence of myopia and high myopia and temporal trends from 2000 through 2050. Ophthalmology. 2016;123:1036-1042. doi:10.1016/j.ophtha.2016.01.006

31. Jonas JB, Pfeil K, Chatzikonstantinou A, Rensch F. Ophthalmodynamometric measurement of central retinal vein pressure as surrogate of intracranial pressure in idiopathic intracranial hypertension. Graef Arch Clin Ophthalmol. 2008;246:1059-1060. doi:10.1007/s00417-008-0780-0

32. Jonas JB, Wang N, Wang YX, et al. Incident retinal vein occlusions and estimated cerebrospinal fluid pressure. The Beijing Eye Study. Acta Ophthalmol. 2015;93:e522-526. doi:10.1111/aos.12575

33. Ren R, Jonas JB, Tian G, et al. Cerebrospinal fluid pressure in glaucoma. A prospective study. Ophthalmology. 2010;117:259-266. doi:10.1016/j.ophtha.2009.06.058

34. Leong DP, Teo KK, Rangarajan S, et al. Prognostic value of grip strength: findings from the Prospective Urban Rural Epidemiology (PURE) study. Lancet. 2015;386:266-273. doi:10.1016/S01406736(14)62000-6

35. Sasso FC, Pafundi PC, Gelso A, et al. High HDL cholesterol: a risk factor for diabetic retinopathy? Findings from NO BLIND study. Diabetes Res Clin Pract. 2019;150:236-244. doi:10.1016/j. diabres.2019.03.028

36. Sasso FC, Pafundi PC, Gelso A, et al. Telemedicine for screening diabetic retinopathy: the NO BLIND Italian multicenter study. Diabetes Metab Res Rev. 2019;35:e3113. doi:10.1002/dmrr.3113

37. Galiero R, Pafundi PC, Nevola R, et al. The importance of telemedicine during COVID-19 pandemic: a focus on diabetic retinopathy. J Diabetes Res. 2020;2020:9036847. doi:10.1155/2020/9036847

\section{Publish your work in this journal}

Diabetes, Metabolic Syndrome and Obesity: Targets and Therapy is an international, peer-reviewed open-access journal committed to the rapid publication of the latest laboratory and clinical findings in the fields of diabetes, metabolic syndrome and obesity research. Original research, review, case reports, hypothesis formation, expert opinion and commentaries are all considered for publication. The manuscript management system is completely online and includes a very quick and fair peer-review system, which is all easy to use. Visit http://www.dovepress.com/testimonials.php to read real quotes from published authors. 